\title{
Wireless Sensing for the Respiratory Activity of Human Beings: Measurements and Wide-band Numerical Analysis
}

\author{
Lorenzo Scalise, ${ }^{1}$ Valter Mariani Primiani, ${ }^{2}$ Paola Russo, ${ }^{2}$ Alfredo De Leo, ${ }^{2}$ \\ Desar Shahu, ${ }^{2}$ and Graziano Cerri ${ }^{2}$ \\ ${ }^{1}$ Dipartimento di Ingegneria Industriale e Scienze Matematiche (DIISM), Università Politecnica delle Marche, \\ Via Brecce Bianche, 60131 Ancona, Italy \\ ${ }^{2}$ Dipartimento di Ingegneria dell'Informazione (DII), Università Politecnica delle Marche, Via Brecce Bianche, \\ 60131 Ancona, Italy
}

Correspondence should be addressed to Paola Russo; paola.russo@univpm.it

Received 5 December 2012; Revised 11 March 2013; Accepted 20 March 2013

Academic Editor: Renato Cicchetti

Copyright (C) 2013 Lorenzo Scalise et al. This is an open access article distributed under the Creative Commons Attribution License, which permits unrestricted use, distribution, and reproduction in any medium, provided the original work is properly cited.

\begin{abstract}
An electromagnetic sensing system for the measurement of the respiratory activity is presented. The aims are to demonstrate the feasibility of the proposed approach and in particular to evaluate the effect on the measured signal of the distance between the subject and the sensing apparatus. Moreover, an electromagnetic model of the system, including the monitored subject, is proposed as a tool to solve the problem of selecting working parameters for system design. The sensing system is based on the measurement of the phase variation of the reflection coefficient caused by the respiratory activity. The phase signal compared with the thorax displacement measured by a reference instrument shows a high correlation $(R=0.97)$ for different subject postures (sitting, standing, and lying) and a reduction of the signal amplitude with the distance $-0.11 \mathrm{~dB} / \mathrm{cm}$ is reported. The numerical simulations performed on a wide-band highlight the frequencies where the method exhibits the highest sensitivity to thorax movements. The sensitivity can be further improved by reducing the antenna beamwidth. Despite the signal amplitude reduction, the proposed system makes it possible to correctly operate at distances up to $2.5 \mathrm{~m}$.
\end{abstract}

\section{Introduction}

Daily clinical practice often requires the monitoring of important physiological parameters of the patient (respiratory and heart rates, arterial pressure, oxygen saturation, etc.) and commonly known as vital signs. From home recovery to intensive care monitoring, vital signs need to be continuously measured and presented to patient carers or used to activate alarms or therapeutic procedures [1]. In order to measure these quantities, the use of invasive procedures based on contact transducers or electrodes [1] is typically required. However, these procedures can limit the duration of monitoring, restrict the patient's movements, cause signal artifacts, and, in some cases, represent a risk for the patient (burns, microshocks, infections, etc.). It is therefore of great interest to explore the possibility to use wireless technologies for the measurement of vital signs that can avoid a direct contact with the patient's body. Some interesting wireless applications have recently been proposed for heart rate monitoring, based on optical methods that enable to precisely assess heart rate variability [2-5] or on electromagnetic waves [6].

Among vital signs, monitoring of respiratory activity and its rate is of particular importance since the cessation of the respiratory function can be a life-threat, especially for patients in critical conditions. This event can build up gradually and, in this case, the continuous monitoring of respiratory acts is fundamental for identifying and/or predicting such a dangerous event [7]. As far as noncontact respiratory rate monitoring is concerned, different approaches have been proposed [8-12]. Such minimally-invasive methods, mainly used for remote monitoring purposes and diagnosis of sleep disorders, are resistive, piezoelectric or inductive thoracic 
belts $[8,9]$, transthoracic impedance transducers [11] or under-mattress pressure mats [12]. The main limitations of such methods are due to movement artifacts and the subject's position (i.e., for pressure mats the subject has to lie on the bed); in particular, the use of chest belts is strongly limited in the case of patients with reduced expired/inspired air exchange and is not applicable for long and medium-term monitoring purposes [8].

As a consequence, the possibility to monitor the respiratory activity with a noncontact method has been an object of numerous recent investigations [13-19]. Laser Doppler vibrometry has demonstrated to be valid and reliable during in-vivo tests [13-15], whereas a second alternative approach is electromagnetic (EM) sensing [16-23]. The first EM approaches proposed $[16,17]$ were not adopted because of the bulky, complex, and rather expensive hardware. With a progressive reduction of dimensions and costs of radiofrequency components and systems, the interest in this sensing technique has rapidly increased. At present, there are two main categories of noncontact vital sign electromagnetic detection systems: ultra-wideband (UWB) pulse radar and continuous wave (CW) Doppler radar. Recent examples of pulsed UWB radar [18-20] were adopted to precisely measure respiration and heartbeat. Echo signals using mono [22] and double antenna [23] CW systems are measured at different working frequencies aiming to detect vital signs. These recent works are of extreme interest in view of a future transfer of this approach to wireless, market-ready monitoring systems. In [24], a novel, wireless, low-cost, and wearable Doppler radar system composed of textile materials and capable of detecting moving objects behind a barrier is presented; the on-body system is capable of detecting multiple moving subjects in indoor environments, including through-wall scenarios.

The majority of these papers demonstrate the possibility to measure a time signal synchronous to the respiratory rhythm and proportional to the variation of the thorax volume caused by inspiration/expiration acts. The great interest on the EM sensing of the respiratory activity is due to the wireless feature of the technique, realized with small antennas illuminating from a distance. Moreover, it is important to underline that, in order to operate, the antenna does not need to be accurately pointed toward a specific part of the subject's body (as for laser-based methods), but it can generally illuminate the region where the monitored subject is positioned [22-25]. Finally, it is worth noting that EM sensing makes it possible to measure through clothes or fabric (bed sheets, blankets, clothes, etc.) without signal disturbances [19]; such a characteristic is a valuable aspect especially in the case of patients who are forced to stay in bed or noncollaborative or unconscious subjects (neonates in incubators, burn-injured, or postoperative patients).

The aim of this paper is therefore to propose an electromagnetic (EM) method for the detection of the respiratory activity and the measurement of the respiratory rate, based on the measurement of the EM signal backscattered by the monitored subject. In particular, the phase variation of the input reflection coefficient of an antenna illuminating the subject during its normal respiratory activity is analyzed in the frequency domain. This approach makes it possible investigate the backscattered signal over a wide frequency range. The availability of a wide band characterization will make it possible to optimally choose the operating frequency if a continuous wave (CW) system is sought or to properly choose the frequency content to build up an optimal pulse if an UWB technology is used. Many parameters contribute to the system performance, and for a good system design, the quantitative assessment of the received signal in dependence on the distance and on the subject posture (standing, sitting, or laying in front of the measurement apparatus) is essential. This is particularly important for the definition of the parameters at the design stage of the system. Such dependences have been investigated using a dedicated experimental set-up able to operate in frequency domain. The EM model was developed and tested to provide the voltage at the input/output port of the transmitting/receiving antenna as a function of the antenna characteristics and of the interaction between the radiated field and the monitored body. In particular, the model made it possible to perform a wide frequency band analysis of the system sensitivity, and a parametric study with respect to the antenna radiating characteristic. The design of an "ad hoc" antenna (i.e., printed antennas $[26,27])$ and systems, characterized by reduced costs and increased sensitivity and portability (i.e., market available system [28]) based on the results obtained in this paper, is left to a future step.

\section{Experimental Set-Up and Testing Conditions}

2.1. Experimental Set-Up. The measurement method used for the detection of the respiratory activity is schematically reported in Figure 1 [22]. A vectorial network analyzer (VNA) (HP 8753D) is used to generate the output signal at $6 \mathrm{GHz}$ $(1 \mathrm{~mW})$ to feed the antenna. The VNA measures the reflection coefficient of the antenna $\left(S_{11}\right)$ which depends on the antenna itself and on the reflected signal backscattered from the body.

The reflection coefficient is a complex quantity, so it is possible to use both amplitude and phase time variations to gain information about the respiratory activity of the subject positioned in front of the antenna. In order to have a sufficiently sensitive system, phase variations were used. In fact, being the thorax displacement $(\Delta D)$ due to the respiratory activity a small percentage of the total distance, the amplitude variation is small. On the other hand, $\Delta D$ is a significant fraction of the wavelength used $(\lambda=5 \mathrm{~cm})$, therefore the variation of the phase of the reflected signal $(\Delta P)$ is easily detectable.

VNA performs a continuous measurement of the reflection coefficient $S_{11}$ (modulus and phase) of the antenna, after a proper calibration procedure, over a time interval that can be chosen by the user (Sweep time). Thanks to this continuous wave (CW) operating modality, the output frequency remains the same during the instrument sweep and therefore the reflection coefficient variations can be ascribed only to the subject's respiratory activity. 


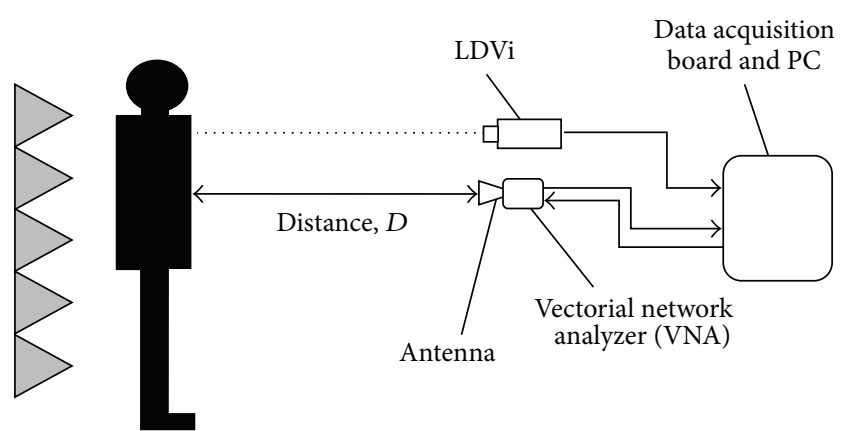

FIGURE 1: Scheme of the experimental set-up.

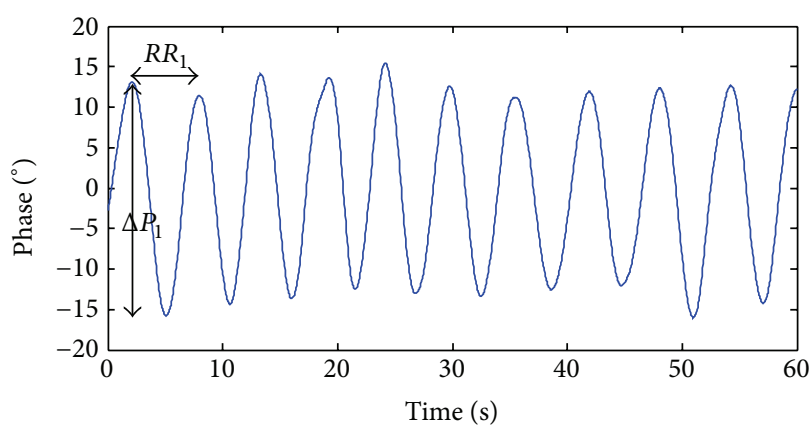

FIGURE 2: Time domain response of the $S_{11}$ phase signal measured at $50 \mathrm{~cm}$ from the subject's thorax during regular respiration (time window $60 \mathrm{~s}) ; \Delta P_{1}$ is the phase variation measured during the first respiratory act, $R R_{1}$ is the 1st respiratory period.

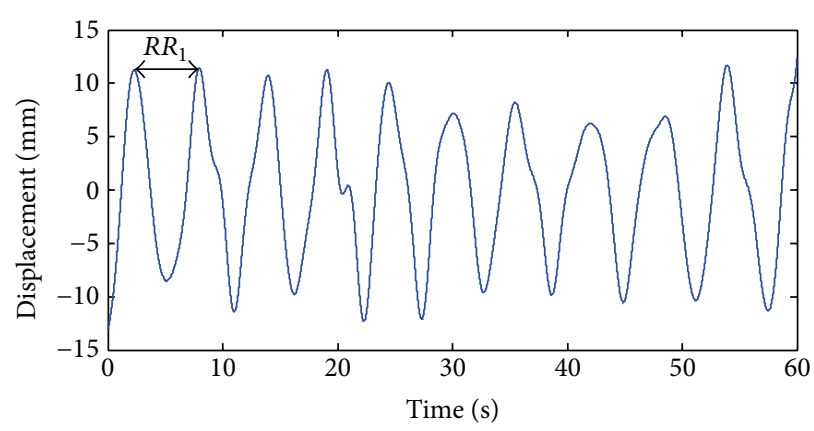

FIgURE 3: Distance variation $\Delta D$ of the subject's thorax (sternum) measured by LDVi at $50 \mathrm{~cm}$ during regular respiration (time window $60 \mathrm{~s}) ; R R_{1}$ is the 1st respiratory period. exhibits a half power beam of about $41^{\circ}$ in $\mathrm{H}$ plane and $66^{\circ}$ in the E plane, at $6 \mathrm{GHz}$.

A laser Doppler vibrometer (LDVi) was used for the measurement of the respiratory acts, as a reference instrument. LDVi was proved [13-15] to accurately provide the oscillations of the skin surface in correspondence to the respiratory acts.

The phase of the $S_{11}$ signal was sampled at $26 \mathrm{~Hz}$ (maximum available data sampling), while the velocity signal from the LDVi was initially sampled at $1 \mathrm{kHz}$. On both signals, the phase of $S_{11}$ measured by the proposed system and the LDVi signal, a wavelet filter (dB8, 5th order for the $S_{11}$ signal and 10th level for the LDVi) was applied in order to reject the disturbances caused by motion artifacts, high frequency noise, and heartbeats [22]. The LDVi velocity signal was integrated to obtain the surface displacement time history. As a final step, the LDVi displacement signal was down-sampled to the same sampling frequency of the $S_{11}$ signal $(26 \mathrm{~Hz})$. Examples of the $S_{11}$ phase and LDVi displacement signals simultaneously measured are reported in Figures 2 and 3, respectively.

In this case, the subject was sitting and normally breathing; the antenna was placed at a distance $D=50 \mathrm{~cm}$. The variation of the displacement due to the respiratory activity is reported on Figure 3, and it is evident how such variation of the displacement produces a variation in the phase of the reflection coefficient simultaneously recorded by the measurement system proposed (Figure 2). It is also possible to note how the operating frequency $(6 \mathrm{GHz})$ exhibits a sufficiently small wavelength $(5 \mathrm{~cm})$ to make thorax excursions (Figure 3) detectable.

2.2. Testing Conditions. We operated our tests on a single male volunteer, aged 22, with a body index mass (BMI, [1]) of $19.59\left[\mathrm{~kg} / \mathrm{m}^{2}\right]$.

The measurement apparatus and the subject were held at 5 measurement distances: 50, 100, 150, 200, and $250 \mathrm{~cm}$. The subject assumed three postures: standing, sitting and lying (on a semirigid bed). While for the standing and sitting postures; the antenna was kept in front of the subject for the laying posture, the subject was asked to assume three positions: supine, prone, and lateral. Each test had a duration of $60 \mathrm{~s}$ and, for each distance and position, it was repeated 3 times. A total of $2880 \mathrm{~s}$ of acquisition time were recorded. During measurement, the subject was asked to breathe normally.

In order to correlate the amplitude of the signal measured by the e.m. system and the distance $D$, we have measured the peak-to-peak phase variation $\overline{\Delta P}$, mean of the $\Delta P_{i}$ values measured on all the 3 tests repeated for each distance in correspondence with the respiratory acts (in Figure 2, the $\Delta P_{1}$ value is reported for the first respiratory act). 


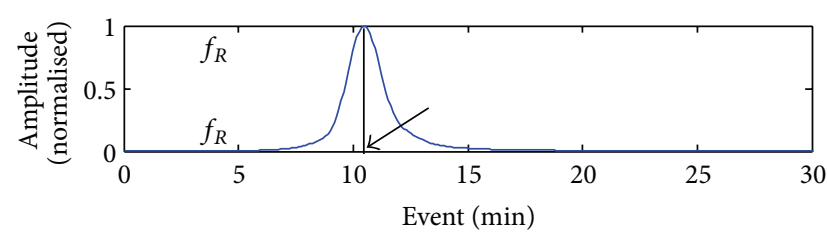

(a)

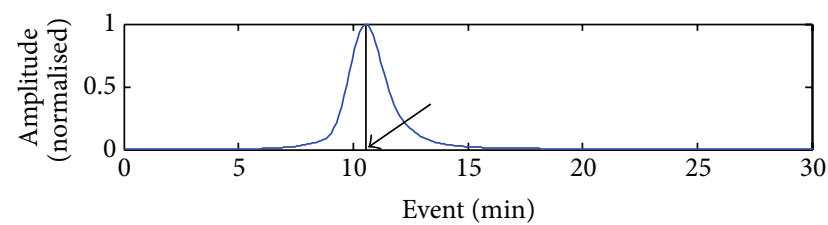

(b)

Figure 4: (a) Power spectrum of $S_{11}$ phase signal (same data of Figure 2). (b) Power spectrum of the displacement signal measured by the LDVi (same data of Figure 3).

As a second parameter describing the quality of the measurement condition, we have defined [22] the quantity signalto-noise ratio (SNR), according to the following formula:

$$
\mathrm{SNR}_{\mathrm{dB}}=20 \log _{10}\left(\frac{\overline{\Delta P}}{V_{n}}\right),
$$

where $\overline{\Delta P}$ is the mean of the $\Delta P$ values measured from the phase of the $S_{11}$ signal (filtered) and $V_{n}$ is the RMS (root mean Square) of the phase of the $S_{11}$ signal (noise), measured without the presence of the subject.

The mean respiratory frequency $\left(f_{R}\right)$ for each test has been calculated for both systems (Figure 4) from the power spectrum of the measured signals (after removal of the DC component).

\section{Results}

The possibility to use the proposed experimental set-up for the detection of the respiratory activity is proved by the trace shown in Figure 5, where a time sequence of 37 s of the $S_{11}$ phase signal is reported for the case of the subject sitting in front of the system at the distance $D$ of $20 \mathrm{~cm}$ (Figure 5). For this demonstrative test, the tidal volume of the subject was simultaneously measured using a reference instrument (spirometer, MLT 1000L ADI), and this is also reported in Figure 5. For this test, the volunteer was asked to follow a specific respiratory rhythm (about $10 \mathrm{~s}$ of high respiration rate, followed by $10 \mathrm{~s}$ of apnea, and finally slow/normal respiratory rhythm).

From Figure 5, it is evident how the $S_{11}$ phase signal is highly correlated with the instantaneous value of the tidal volume simultaneously measured by the spirometer. The $\overline{\Delta P}$ value (mean and standard deviation) measured for each distance $D$ tested is illustrated in Figure 6 for the three postures: sitting, standing, and supine (only at $D=100 \mathrm{~cm}$ and $D=150 \mathrm{~cm}$ ). Some signal spikes, due to rapid movement artifacts, are visible on the first part of $S_{11}$ phase signal;

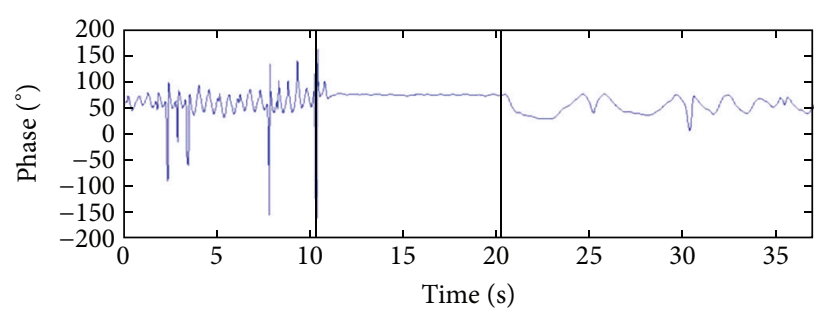

(a)

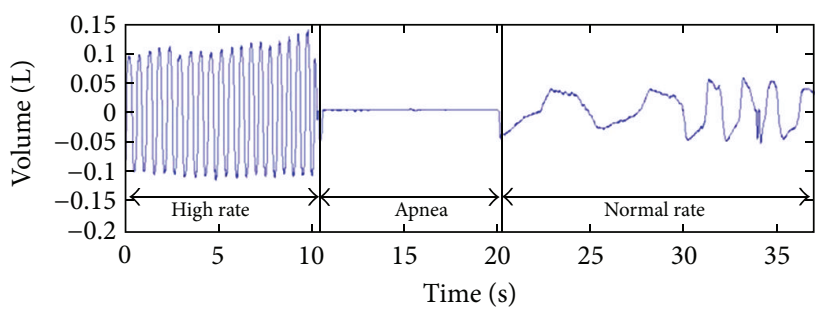

(b)

Figure 5: Comparison of the $S_{11}$ phase signal (a) and spirometer signal (b) subject sitting, distance $D=20 \mathrm{~cm}$.

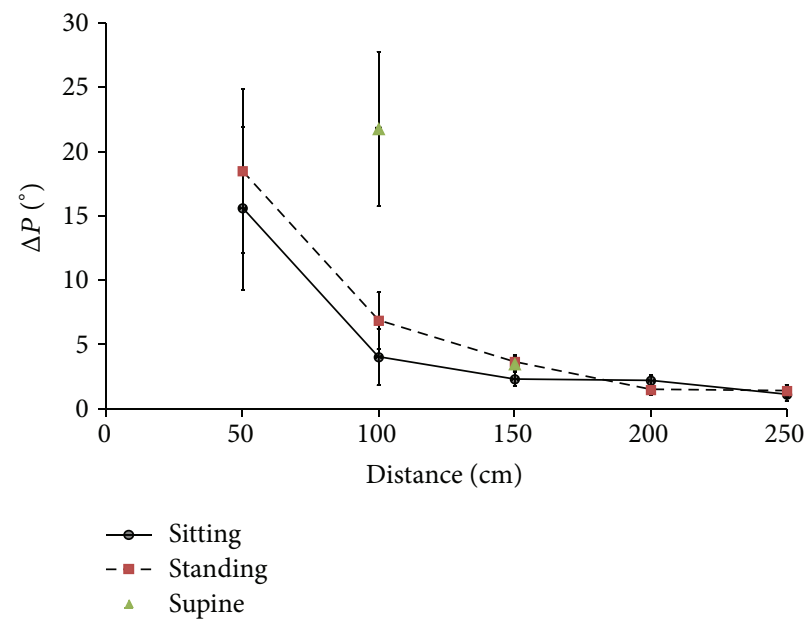

FIgUre 6: Mean and standard deviation of $\Delta P$ in function of the distance $D$ for sitting, standing, and lying postures.

the same phenomena are not detected by the spirometer measuring the subject air flow.

From the results obtained, it is possible to observe that, for both the postures, there is a reduction of $\overline{\Delta P}$ as the distance $D$ is increased.

The system exhibits the maximum phase variation for the closest distances $\left(19^{\circ}\right.$ at $50 \mathrm{~cm}$, standing) while, even still detectable, a minimum phase variation is reported for the longer distances tested $\left(1^{\circ}\right.$ at $\left.250 \mathrm{~cm}\right)$. It is also possible to note how, for all the distances $D$, the measured data are all compatible, highlighting the absence of the dependence of $\overline{\Delta P}$ on the posture. The only exception is reported for the supine posture at $100 \mathrm{~cm}$ where $\overline{\Delta P}$ reaches the highest value measured $\left(21^{\circ}\right)$; this is probably due to the fact that the supine posture (the subject was lying on a semi-rigid 


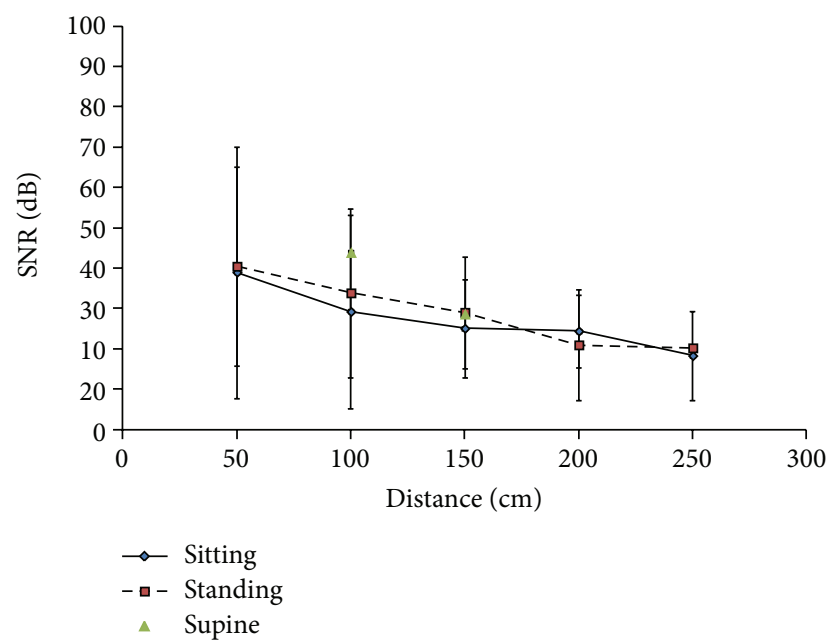

Figure 7: SNRs values (mean and standard deviation) in the function of $D$ and for each of the postures.

bed) accentuated the thorax excursion during the normal respiratory acts.

From Figure 6, it is also possible to observe the $\overline{\Delta P}$ reduction of $74 \%$ and $63 \%$ between the data measured at $50 \mathrm{~cm}$ and the data measured at $100 \mathrm{~cm}$, for sitting and standing postures, respectively. This signal reduction is due to the fact that the radiated beam for $D>80 \mathrm{~cm}$, according to the radiating antenna pattern characteristics, is no longer focused exclusively on the subject thorax, but some of the incident power is also transmitted to the background, and it does not contribute to the generation of the reflected signal.

The SNRs of the signals measured, calculated according to (1), are shown in Figure 7 for the tested postures.

As for $\overline{\Delta P}$, it is evident how the greater $D$, the lower SNR, and this is confirmed for all the postures. The highest value $(39 \mathrm{~dB})$ is obtained at the distance of $50 \mathrm{~cm}$ for the standing posture, while the lowest $(18 \mathrm{~dB})$ is reported when the subject is sitting at $250 \mathrm{~cm}$. It is also possible to note how the SNRs values are compatible for all the distances, confirming that posture is not a significant parameter.

From the data measured, it is possible to estimate an SNR loss of $-0.11 \mathrm{~dB} / \mathrm{cm}$ when the subject is standing in front of the antenna and of $-0.9 \mathrm{~dB} / \mathrm{cm}$ when the subject is sitting. Such values make it possible to estimate an SNR reduction of more than $27 \mathrm{~dB}$ for distances $>3 \mathrm{~m}$, independently from the subject's posture (sitting or standing).

In Figure 8, the scatter plot of the mean respiratory frequencies $\left(f_{R}\right)$ measured by the LDV and the proposed system, together with the fitting line (mean square), is shown for standing, sitting, and supine postures. In this case, the correlation coefficient calculated between the two systems is 0.97 .

Finally in Figure 9, we illustrate the case of the subject lying $(D=100 \mathrm{~cm})$ and assuming 3 positions: supine, prone and lateral. Also in this case, a good value for the correlation coefficients calculated between the two systems is verified: 0.94, 0.93, and 0.98 for supine, prone, and lateral positions, respectively.

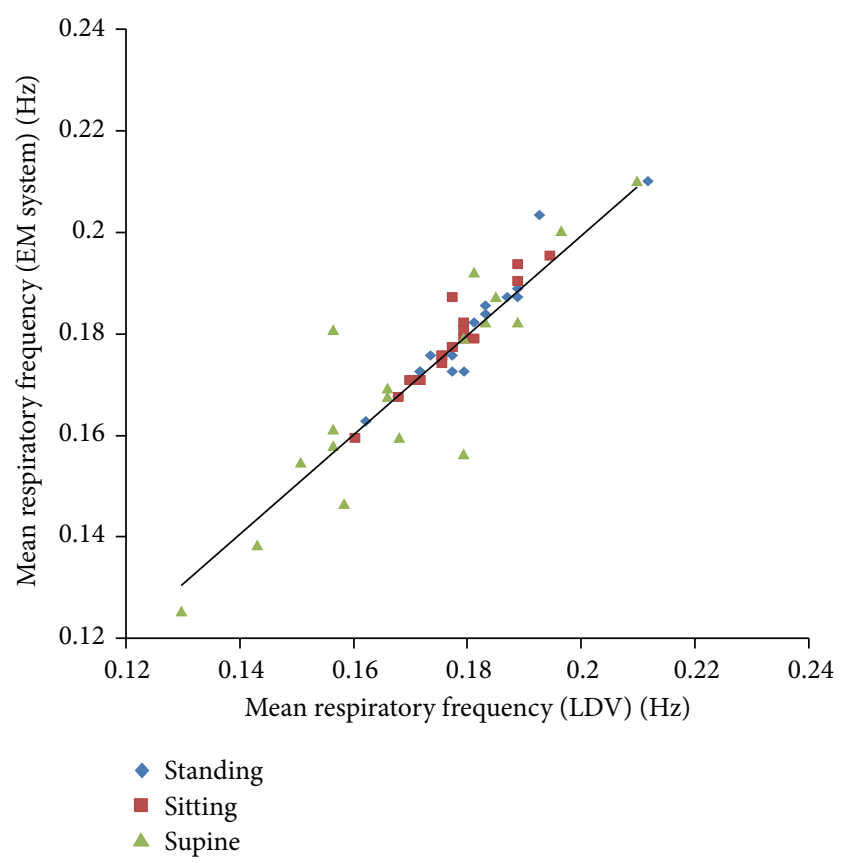

FIGURE 8: Scatter plot of mean respiratory frequencies $\left(f_{R}\right)$ : LDVi versus proposed method (subject position: standing, sitting, and supine).

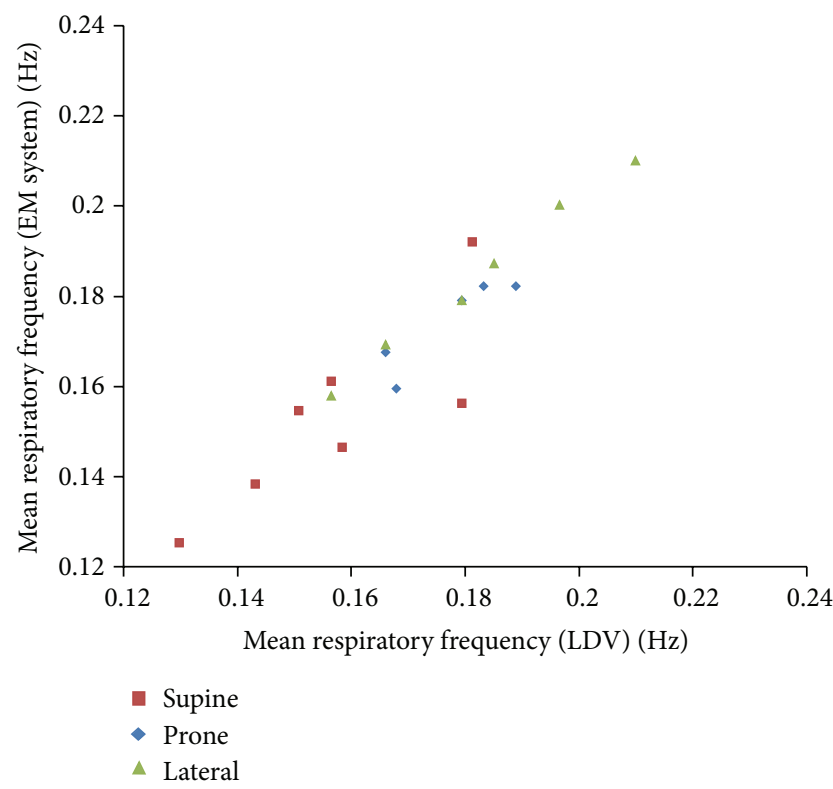

FIGURE 9: Scatter plot of mean respiratory frequencies $\left(f_{R}\right)$ : LDVi versus proposed method (supine, prone, and lateral; $D=100 \mathrm{~cm}$ ).

\section{Electromagnetic Model for Wide Band Analysis}

An electromagnetic model of the interaction between the monitored subject and the electromagnetic system is proposed. The aim is to provide a characterization of the field scattered by the thorax of a subject during its normal 


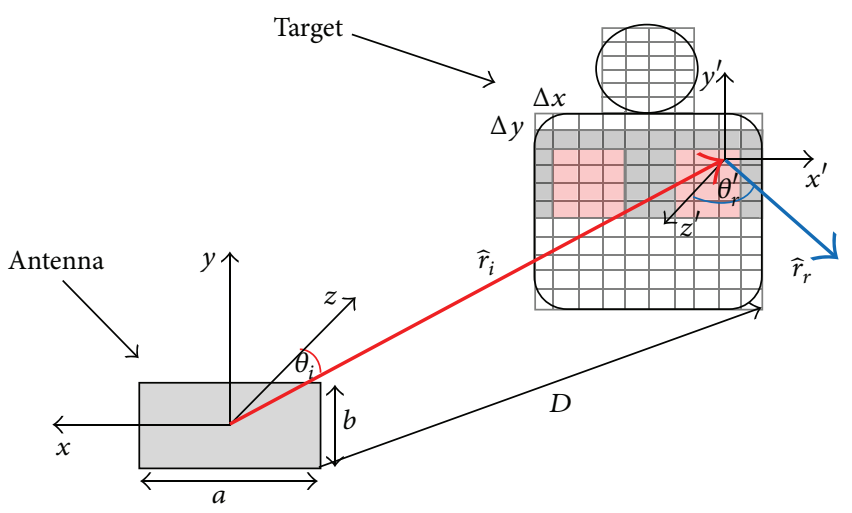

FIgURE 10: Geometry of the antenna and the target.

respiratory activity and to evaluate variations of the received signal $\left(S_{11}\right)$ in a wide frequency band $(2-12 \mathrm{GHz})$.

More in general, the model proposed can be used to analyze the influence of some important parameters (working frequency, antenna field pattern, etc.) on amplitude and phase variations of the measured signal $\Delta P$ (sensitivity) with the goal of optimizing the future design of e.m. systems using the same working principle.

Figure 10 illustrates the geometry of the scenario of interest which is composed by an antenna radiating toward a target placed at a distance $D$. The target is a simplified representation of a human thorax, where a subarea of the total target (colored region in Figure 10) may change its relative position along the $z$ direction with respect to the remaining part of the target (the white region in the figure). In particular, to highlight the effect on the phase variation of the complex movement of an actual thorax, two different areas have been introduced to characterize larger and smaller amplitude of displacement (red and gray areas in Figure 10, resp.). The antenna is an equivalent ideal aperture antenna, having a radiation pattern with the same beamwidth of the actual antenna. The aperture has a uniform $y$-directed field distribution $E_{0}$, and its dimensions $(a \times b)$ are chosen so to provide the desired half power beamwidth $\Delta \theta$ and $\Delta \varphi$ in the $\mathrm{E}$ and $\mathrm{H}$ planes, respectively (as reported later).

The dominant $y$-component of the incident electric field, radiated by the aperture in the direction $\widehat{r}_{i}$ (referred to a spherical system associated to the Cartesian system $x, y, z$ ) and assuming far field conditions is given by [25]

$$
E_{y}^{i}=j \frac{a b E_{0} e^{-j\left(2 \pi r_{i} / \lambda\right)}}{\lambda r_{i}} \cos \left(\theta_{i}\right) \frac{\sin \left(X_{i}\right)}{X_{i}} \frac{\sin \left(Y_{i}\right)}{Y_{i}},
$$

where:

$$
\begin{aligned}
X_{i} & =\frac{\pi a}{\lambda} \sin \left(\theta_{i}\right) \cos \left(\varphi_{i}\right), \\
Y_{i} & =\frac{\pi b}{\lambda} \sin \left(\theta_{i}\right) \sin \left(\varphi_{i}\right) .
\end{aligned}
$$

$r_{i} \theta_{i}, \varphi_{i}$ are the coordinates of the observation point in the spherical system centered on the aperture.

The field radiated by the aperture impinges on the body's surface from the incident direction $\widehat{r}_{i}$ and is partially reflected in the direction $\widehat{r}_{r}$, as shown in Figure 10. The received signal $\left(S_{11}\right)$ is calculated evaluating the electric field coupled back with the antenna.

In this simplified model, to account for the amplitude and phase variations of the incident field, the body surface facing the antenna is subdivided in $n$ subareas $(n=134)$, and each subarea is considered as a radiating aperture with a uniform field distribution. The reflected electric field is calculated considering the oblique incidence of a plane wave on a dielectric interface, and its value is

$$
E_{y}^{-}=\rho_{\mathrm{TM}} E_{\theta_{i}}^{i} \cos \theta_{r}^{\prime} \sin \varphi_{r}^{\prime}-\rho_{\mathrm{TE}} E_{\varphi_{i}}^{i} \cos \varphi_{r}^{\prime},
$$

where $\theta_{r}^{\prime}, \varphi_{r}^{\prime}$ define the direction of the reflected wave in the local coordinate system $\left(x^{\prime}, y^{\prime}, z^{\prime}\right)$ centered in the $n$th subarea, $\rho_{\mathrm{TM}}, \rho_{\mathrm{TE}}$ are the reflection coefficients of the transverse components of the electric field on the incidence plane [25] and depend on the incidence angle and on the dielectric properties of the body; $E_{\theta_{i}}^{i}$ and $E_{\varphi_{i}}^{i}$ are the incident electric field components in spherical coordinate system.

Similarly to physical optic approach, the interaction among the fields of adjacent subareas is assumed to be negligible. The subarea dimensions $\Delta x$ and $\Delta y$ are chosen so that the transmitting aperture antenna can be considered in the far field zone of the radiating $n$th element; the $E_{y}$ field component radiated by this element is

$$
E_{n y}=j \frac{\Delta x \Delta y E_{y}^{-} e^{-j\left(2 \pi r_{r} / \lambda\right)}}{\lambda r_{r}} \cos \left(\theta^{\prime}\right) \frac{\sin \left(X_{n}\right)}{X_{n}} \frac{\sin \left(Y_{n}\right)}{Y_{n}},
$$

where

$$
\begin{gathered}
X_{n}=\frac{\pi \Delta x}{\lambda}\left[\sin \left(\theta^{\prime}\right) \cos \left(\varphi^{\prime}\right)-\sin \left(\theta_{r}^{\prime}\right) \cos \left(\varphi_{r}^{\prime}\right)\right], \\
Y_{n}=\frac{\pi \Delta y}{\lambda}\left[\sin \left(\theta^{\prime}\right) \sin \left(\varphi^{\prime}\right)-\sin \left(\theta_{r}^{\prime}\right) \sin \left(\varphi_{r}^{\prime}\right)\right],
\end{gathered}
$$

being $r^{\prime}, \theta^{\prime}, \varphi^{\prime}$ the spherical coordinates of the observation point expressed in a local coordinate system $\left(x^{\prime}, y^{\prime}, z^{\prime}\right)$ centred in the $n$th subarea, and $\theta_{r}^{\prime}, \varphi_{r}^{\prime}$ the direction of the reflected wave. The total field scattered by the body and received by the antenna is

$$
E_{y}^{\mathrm{sc}}=\sum_{n=1}^{N} E_{n y} .
$$

The knowledge of the signal received by the antenna makes it possible to evaluate the $S_{11}$ scattering parameters of the antenna:

$$
S_{11}=\Gamma_{\text {ant }}+\Gamma_{\text {sc }} \sqrt{\frac{\left(1-\left|\Gamma_{\text {ant }}\right|^{2}\right) \eta}{Z_{0} a b}} \vec{l}_{\text {eff }} \cdot \hat{y} \frac{Z_{0}}{Z_{\text {ant }}+Z_{0}},
$$

where $\Gamma_{\text {ant }}$ and $Z_{\text {ant }}$ are, respectively, the reflection coefficient and the input impedance of the antenna radiating in free space, $Z_{0}$ is the characteristic impedance of the line feeding the antenna, $\eta$ is the free space wave impedance, and $l_{\mathrm{eff}}$ is the effective length of the antenna, $\Gamma_{\mathrm{sc}}=E_{y}^{\mathrm{sc}} / E_{0}$. 


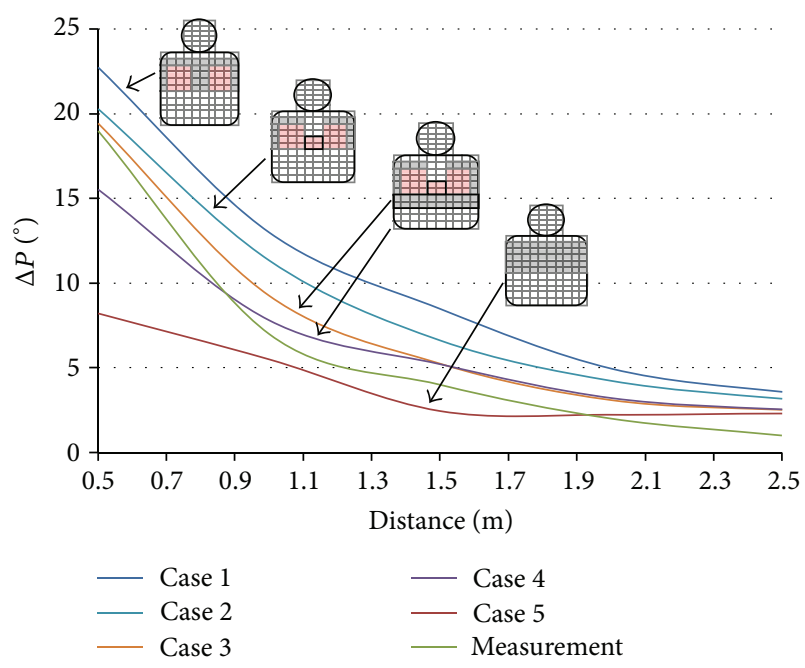

Figure 11: Maximum phase variation for different type of breathing movements simulated and measured.

The model results were applied using the same testing conditions used for the measurements of Figure 6. The antenna used for the measurements has $\Gamma_{\text {ant }}=0.062+0.19 i$ at the working frequency $f=6 \mathrm{GHz}$. The data implemented for the model are $\Delta x=\Delta y=5 \mathrm{~cm}$; conductivity of the body $\sigma_{\text {body }}$ $=1$; relative permittivity of the body $\varepsilon_{\text {body }}=70$; half power beamwidth: $\Delta \theta=66^{\circ}, \Delta \varphi=41^{\circ}$ (from which the aperture dimensions are derived: $a=\lambda / 0.79, b=\lambda / 1.229)$. The distance between the aperture antenna and the colored subareas of Figure 10 was changed in a few steps as: $D-k \Delta d$, where $\Delta d$ $=1 \mathrm{~mm}$ for the gray area and $\Delta d=2 \mathrm{~mm}$ for the red area of Figure 10, and $k=0.10$. The chest displacement chosen is typical for human breathing (see e.g., Figure 3). In Figure 11, the results obtained with the model and the measurement of Figure 6 (standing) are reported. In the same figure, it is possible to see the model results obtained changing the breathing behavior of the subject. In particular, for case 1, 2, 3 , and 5 the grey zones and the red zones have, respectively, a maximum displacement of $1 \mathrm{~cm}$, and $2 \mathrm{~cm}$, whereas for case 4 the grey and the red zones have respectively a maximum displacement of $1 \mathrm{~cm}$ and $1.8 \mathrm{~cm}$.

Figure 11 shows how the complexity of the mechanism involved in the respiration acts affects the electromagnetic results. The phase variation depends strongly on the way of breathing, that is, on the position variations of the points on the thorax surface. Moreover, these variations are differently detected by the antenna because it weights the surface movements with its radiation pattern. From the same figure it is possible to see that the model is very sensitive to the different thorax movements, and it predicts correctly the order of magnitude of the phase variations due to breathing. A direct comparison of the measurements and the model results would require a precise knowledge of the displacement of each point of the thorax of the subject under measurement.

The proposed model allows to investigate the influence of the frequency, and the radiation beamwidth of the antenna has been carried out.

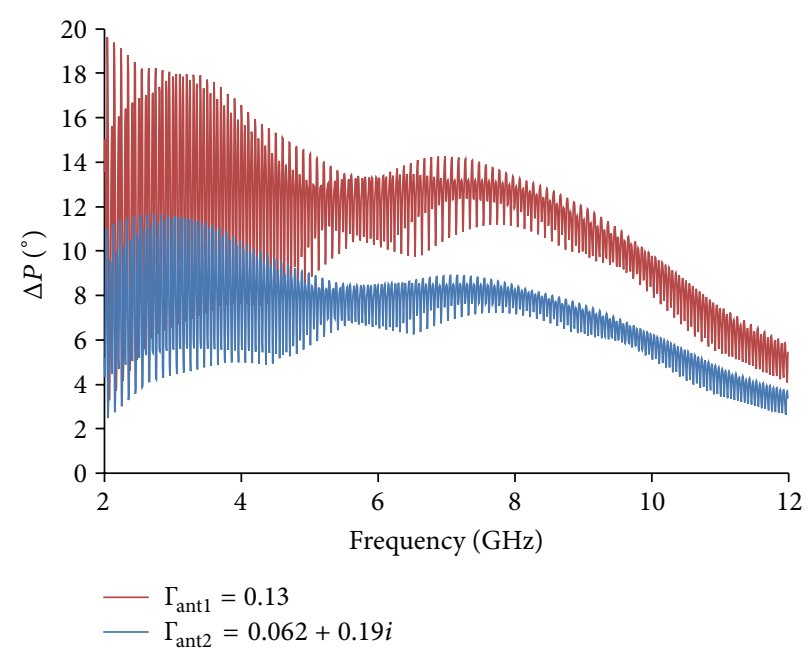

Figure 12: Phase variation (numerical model) as function of the emitted frequency and for two antenna reflection coefficients.

Considering the subject placed at a distance $D$ of $150 \mathrm{~cm}$, with the numerical model (using case 1), it is possible to infer the phase variations due to the respiratory acts at different working frequencies in the interval $2-12 \mathrm{GHz}$, maintaining the other experimental parameters as described previously. In Figure 12, we report the phase variation at a target distance $D$ of $150 \mathrm{~cm}$ in function of the emitted frequency and for two antenna matching conditions:

$$
\Gamma_{\text {ant1 }}=0.13, \quad \Gamma_{\text {ant2 }}=0.062+0.19 i .
$$

The maximum phase variation exhibits an oscillating behavior with a period that depends on the distance between the antenna and the target. This behavior depends on how the reflected signal and the antenna reflection coefficient combine at different frequencies. Figure 13 shows the $S_{11}$ on the complex plane during a respiratory act for 5 different frequencies. These frequencies are chosen in the range of the highest variations. It is clearly evident that the two components in (8) combine differently and that, in particular, there are two frequencies $(2.72$ and $2.78 \mathrm{GHz})$ that have a larger phase variation then the others.

Figure 12 also shows that the phase $\Delta P$ depends on the frequency and that there are some frequencies where it can reach a maximum value; therefore, these frequencies can be chosen to obtain a more sensitive system. The same figure also shows the phase variation for two different antenna reflection coefficients. It is interesting to note that the matching of the antenna plays an important role in the value of the phase variation amplitude $\Delta P$ and that the higher the impedance matching, the greater is the phase variation. This behavior is due to the signal backscattered by the antenna itself, which combines together with the signal coming from the target masking the information on respiratory movements.

A similar analysis can be conducted modifying the directivity of the antenna (beamwidth). We have kept all the test parameters equal to the ones used for case 1 of Figure 11, but three different half power beamwidths are considered: 


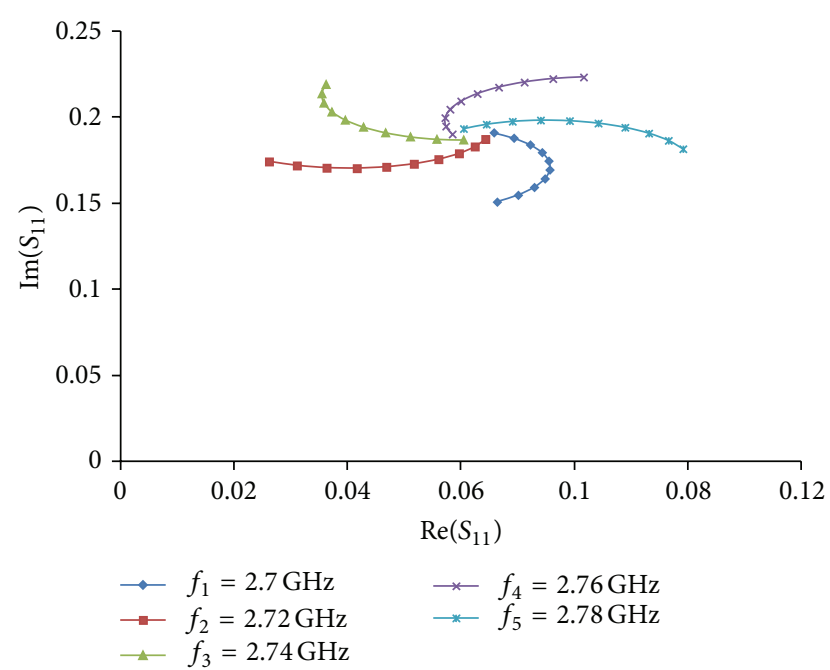

FIGURE 13: $S_{11}$ at the input port of the antenna in the complex plane for 5 different frequencies.

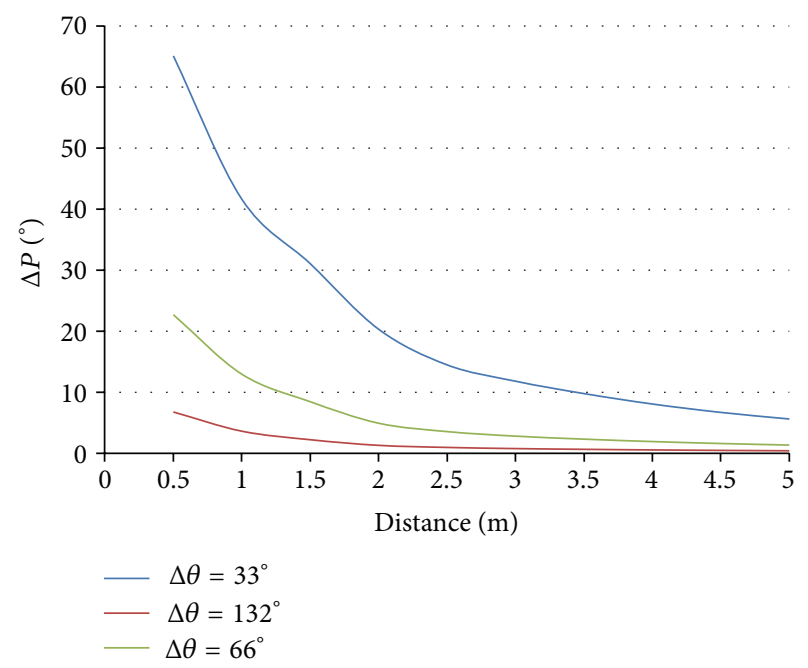

FIGURE 14: Effect of the half power beamwidth $(\Delta \theta \Delta \phi)$ on the phase variation for increasing distances $D$.

(1) $\Delta \theta=66^{\circ}, \Delta \varphi=41^{\circ}$ (2) $\Delta \theta=33, \Delta \varphi=20$, (3) $\Delta \theta=132^{\circ}$, and $\Delta \varphi=82^{\circ}$.

In Figure 14, the phase variation of the received signal for the three cases is illustrated, when the target is placed at different distances.

It can be seen that the beamwidth greatly affects the sensitivity of the system, in particular when the target is placed near the antenna. This result is due to the focalization effect of the increasing directivity which increases the reflected signal level because most of the radiated power is intercepted by the moving part of the target.

It is also possible to observe that, with the target placed at a distance of $150 \mathrm{~cm}$ from the antenna, the phase variation of the reflected signal for $\Delta \theta=33^{\circ}$ is considerably greater with respect to the case of $\Delta \theta=66^{\circ}$. This effect is not so evident away from the antenna, where the free space attenuation of the signal is increased and where, for all the three cases, the target is partially radiated by the incident power.

As a consequence, it is possible to note that while an increase in frequency does not significantly influence the value of the detected phase variations caused by respiratory activity, the reduction of the antenna beamwidth appears to positively influence the phase of the signal detected $\left(S_{11}\right)$ during respiration.

\section{Discussion and Conclusions}

A wireless noncontact approach to the problem of the measuring of the respiratory activity of human subjects has been proposed, using an electromagnetic sensing system. This method is based on the measurement of the phase variations of the reflection coefficient $S_{11}$ caused by the displacement of the thorax surface, and an electromagnetic model has been proposed to reproduce the system response. The model is based on a frequency domain approach, and it is an useful tool for investigating the influence of the parameters on the system performances.

The proposed method is not only limited to a CW monitoring, as it has been demonstrated by the wideband frequency domain analysis carried out with the electromagnetic model. In fact, useful information about the design of an UWB pulse monitoring system can be also easily achieved from the obtained results. For example, from the results shown in the previous section, it can be inferred that the most suitable pulse for a pulse monitoring system should have the significant part of its spectrum in the range $2-4 \mathrm{GHz}$ to maximize sensitivity or the range $4-6 \mathrm{GHz}$ for a flat response.

The solution proposed is feasible for brief as well as for prolonged observation time which is the main limitation of the systems available for respiration monitoring $[8,20]$. Change of the respiration frequencies as well as apneas can be clearly detected (Figure 5$)$. Good correlations $(R=0.97)$ with the gold standards are also reported for the measured mean respiration frequencies.

The measurement system proposed presents also a reduced sensitivity to the subject posture. Standing, sitting, and laying postures have been investigated and they guarantee good correlations $(R=0.94,0.93$, and 0.98$)$ also for different laying positions (supine, prone, and lateral), meaning that, when lying, the subject observed is free to assume any position during the test, while this is not possible for currently available measurement systems $[8,20]$ which have, in fact, this strong limitation.

In detail, the experimental investigations have highlighted the dependence of the signal amplitude (phase variation in ${ }^{\circ}$ ) on target distance. The $74 \%$ of signal reduction has been reported when the subject was measured at $1 \mathrm{~m}$, with respect to the case of the subject standing at $0.5 \mathrm{~m}$. While for longer distances the signal amplitude is always $<5^{\circ}$. More in detail, we have explored the dependence of the quality of the measured (in particular the signal SNR) on the distance between antenna and subject, $D$. A reduction in the SNR with distance $D$ has been reported (in the order of $-0.11 \mathrm{~dB} / \mathrm{cm}$ ). From our tests, it is shown that despite the fact 
that at $2.5 \mathrm{~m}$ the SNR is reduced (about the 50\%) in respect to the signal measured at $25 \mathrm{~cm}$, it is still sufficiently high to allow a correct detection of the respiratory activity. In the case where a minimum operating SNR is requested, a possible solution could be to improve the emitted power $(1 \mathrm{~mW}$ in our case) or to use a narrower radiating beam for the antenna. Although the quality of the signal can be reduced in some conditions (longer distances), the SNR is still sufficiently high $(>18 \mathrm{~dB})$ to provide reliable data.

The possible implementation of the proposed measurement approach could make use of market ready technologies which are not designed for laboratory use and are available at lower costs [28]. In general such devices are not optimized for the scopes considered in this paper (i.e., measurement of respiratory rate), and therefore they could be improved by the results reported.

Testing in "neutral" laboratory conditions (presence of e.m. absorbers), surrounding the subject under test, is a limitation of our study, and in this preliminary work it has been decided to better focus on the phenomenon, reducing the effect of interfering inputs such as the clutter due to environmental conditions. For future applications, the electromagnetic scattering of the surroundings and in general the clutter received at the antenna need to be analyzed. Moreover, for longer time exposure (some hours), automatic recalibration of the system could be introduced to reduce the effect of electronic circuit drift. In practice, in the case of an hospitalized patient, most of the received signal can be considered as static clutter, and signals variations are only due to the respiratory activity. In more complex situations a dedicated postprocessing will be needed to discriminate between patient's respiration from other nonstatic reflections. This aspect will be taken into account under consideration in our future research activity as well as the need for specifically designed antenna providing precisely shaped radiation patterns.

The method proposed in this paper has been tested only on one motionless person; if two or more subjects will be simultaneously illuminated by the antenna, the sensor will detect a composition of their signals. This can be a limitation of the method investigated, and attempts to resolve this problem have been recently proposed by placing more than one sensor and therefore using spatial diversity [27].

A possible future aspect to be investigated is related to the fact that, from a clinical point of view, "respiratory activity" also includes other aspects such as the depth (flow, volume) of the single air acts. The possibility to calibrate the thorax displacement measured by a laser Doppler vibrometer to the flow or volume exchanged during respiration has been demonstrated [14]. This possibility has been recently explored also for microwave sensors [29]. Such possibility could be studied also for the here proposed em approach.

Different applications of electromagnetic noncontact sensing of human beings can also be considered in the fields of security, rescue in emergency situations, elderly remote care, and so forth. Such perspective has aroused the attention of the scientific community on the potentiality of these kinds of sensors.

\section{Acknowledgments}

The authors would like to thank Dr. Paolo Marchionni and Dr. Ilaria Ercoli for their help in the laboratory sessions.

\section{References}

[1] J. G. Webster, Medical Instrumentation Application and Design, John Willey \& Sons, Boston, Mass, USA, 1992.

[2] L. Scalise and U. Morbiducci, "Non-contact cardiac monitoring from carotid artery using optical vibrocardiography," Medical Engineering and Physics, vol. 30, no. 4, pp. 490-497, 2008.

[3] U. Morbiducci, L. Scalise, M. De Melis, and M. Grigioni, "Optical vibrocardiography: a novel tool for the optical monitoring of cardiac activity," Annals of Biomedical Engineering, vol. 35, no. 1, pp. 45-58, 2007.

[4] M. De Melis, U. Morbiducci, and L. Scalise, "Identification of cardiac events by optical vibrocardiograpy: comparison with phonocardiography," in Proceedings of the 29th Annual International Conference of IEEE-EMBS, Engineering in Medicine and Biology Society (EMBC '07), pp. 2956-2959, August 2007.

[5] M. De Melis, U. Morbiducci, L. Scalise et al., "A preliminary study for the evaluation of large artery stiffness: a non contact approach," Artery Research, vol. 2, no. 3, pp. 100-101, 2008.

[6] S. Bakhtiari, T. W. Elmer, N. M. Cox et al., "Compact millimeterwave sensor for remote monitoring of vital signs," IEEE Transactions on Instrumentation and Measurement, vol. 61, no. 3, pp. 830-841, 2011.

[7] M. I. Bierman, "Respiratory monitoring," in Comprehensive Respiratory Care, D. E. Dantzkner, N. R. Macintyre, and E. D. Bakow, Eds., W. B. Saunders Company, Philadelphia, Pa, USA, 1995.

[8] M. Folke, L. Cernerud, M. Ekström, and B. Hök, "Critical review of non-invasive respiratory monitoring in medical care," Medical and Biological Engineering and Computing, vol. 41, no. 4, pp. 377-383, 2003.

[9] X. Zhu, W. Chen, T. Nemoto et al., "Real-time monitoring of respiration rhythm and pulse rate during sleep," IEEE Transactions on Biomedical Engineering, vol. 53, no. 12, pp. $2553-$ 2563, 2006.

[10] K. Watanabe, T. Watanabe, H. Watanabe, H. Ando, T. Ishikawa, and K. Kobayashi, "Noninvasive measurement of heartbeat, respiration, snoring and body movements of a subject in bed via a pneumatic method," IEEE Transactions on Biomedical Engineering, vol. 52, no. 12, pp. 2100-2107, 2005.

[11] K. P. Cohen, W. M. Ladd, D. M. Beams et al., "Comparison of impedance and inductance ventilation sensors on adults during breathing, motion, and simulated airway obstruction," IEEE Transactions on Biomedical Engineering, vol. 44, no. 7, pp. 555566, 1997.

[12] D. I. Townsend, M. Holtzman, R. Goubran, M. Frize, and F. Knoefel, "Measurement of torso movement with delay mapping using an unobtrusive pressure-sensor array," IEEE Transactions on Instrumentation and Measurement, vol. 60, no. 5, pp. 17511760, 2011.

[13] L. Scalise, P. Marchionni, and I. Ercoli, "Optical method for measurement of respiration rate," in Proceedings of the IEEE International Workshop on Medical Measurements and Applications (MeMeA '10), pp. 19-22, May 2010.

[14] L. Scalise, P. Marchionni, and I. Ercoli, "A non contact, optical procedure for precise measurement of respiration rate and flow," 
in Biophotonics: Photonic Solutions for Better Health Care II, vol. 77150 of Proceedings of SPIE, April 2010.

[15] L. Scalise, I. Ercoli, P. Marchionni, and E. P. Tomasini, "Measurement of respiration rate in preterm infants by laser Doppler vibrometry," in Proceedings of the IEEE International Workshop on Medical Measurement and Applications (MeMeA '11), pp. 657-661, 2011.

[16] J. C. Lin, "Non-invasive microwave measurement of respiration," Proceedings of the IEEE, vol. 63, no. 10, p. 1530, 1975.

[17] E. Greneker, "Radar sensing of heartbeat and respiration at a distance with applications of the technology," Radar, vol. 97, pp. 150-154, 1997.

[18] J. C. Lin, "Microwave sensing of physiological movement and volume change: a review," Bioelectromagnetics, vol. 13, no. 6, pp. 557-565, 1992.

[19] C. Gu, C. Li, J. Lin, J. Long, J. Huangfu, and L. Ran, “Instrumentbased noncontact doppler radar vital sign detection system using heterodyne digital quadrature demodulation architecture," IEEE Transactions on Instrumentation and Measurement, vol. 59, no. 6, pp. 1580-1588, 2010.

[20] J. C. Y. Lai, Y. Xu, E. Gunawan et al., "Wireless sensing of human respiratory parameters by low-power ultrawideband impulse radio radar," IEEE Transactions on Instrumentation and Measurement, vol. 60, no. 3, pp. 928-938, 2011.

[21] C. Li, J. Ling, J. Li, and J. Lin, "Accurate doppler radar noncontact vital sign detection using the RELAX algorithm," IEEE Transactions on Instrumentation and Measurement, vol. 59, no. 3, pp. 687-695, 2010.

[22] L. Scalise, A. De Leo, V. Mariani Primiani, P. Russo, D. Shahu, and G. Cerri, "Non contact monitoring of the respiration activity by electromagnetic sensing," in Proceedings of the IEEE International Workshop on Medical Measurement and Applications (MeMeA '11), pp. 418-422, 2011.

[23] F. Soldovieri, I. Catapano, L. Crocco, L. N. Anishchenko, and S. I. Ivashov, "A feasibility Study for Life SignsMonitoring via a Continuous-Wave Radar," International Journal on Antenna and Propagation, vol. 2012, Article ID 420178, 5 pages, 2012.

[24] S. Agneessens, P. Van Torre, F. Declercq et al., "Design of a wearable, low-cost, through-wall doppler radar system," International Journal on Antenna and Propagation, vol. 2012, Article ID 840924, 9 pages, 2012.

[25] C. A. Balanis, Antenna Theory: Analysis and Design, John Wiley \& Sons, New York, NY, USA, 2005.

[26] P. Bernardi, R. Cicchetti, S. Pisa, E. Pittella, E. Piuzzi, and O. Testa, "Design and realization of a UWB radar for breath activity monitoring," in Proceedings of the International Symposium on Electromagnetic Compatibility (EMC EUROPE '12), pp. 1-6, 2012.

[27] L. W. Chua, "A new UWB antenna with excellent time domain characteristics," in Proceedings of the 8th European Conference on Wireless Technology, pp. 551-554, October 2005.

[28] https://www.novelda.no/content/development-kits.

[29] D. Dei, G. Grazzini, G. Luzi et al., "Non-contact detection of breathing using a microwave sensor," Sensors, vol. 9, no. 4, pp. 2574-2585, 2009. 

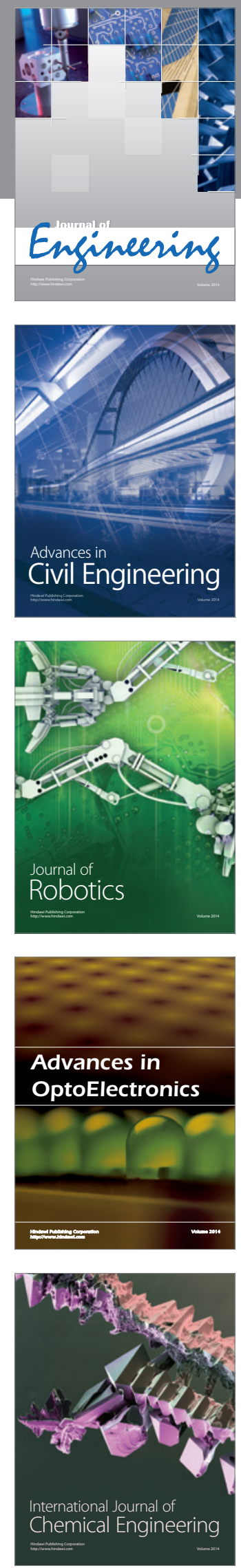

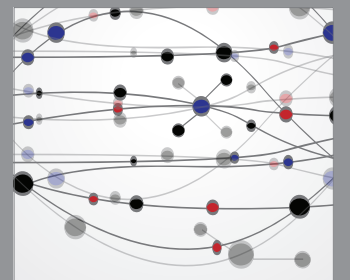

The Scientific World Journal
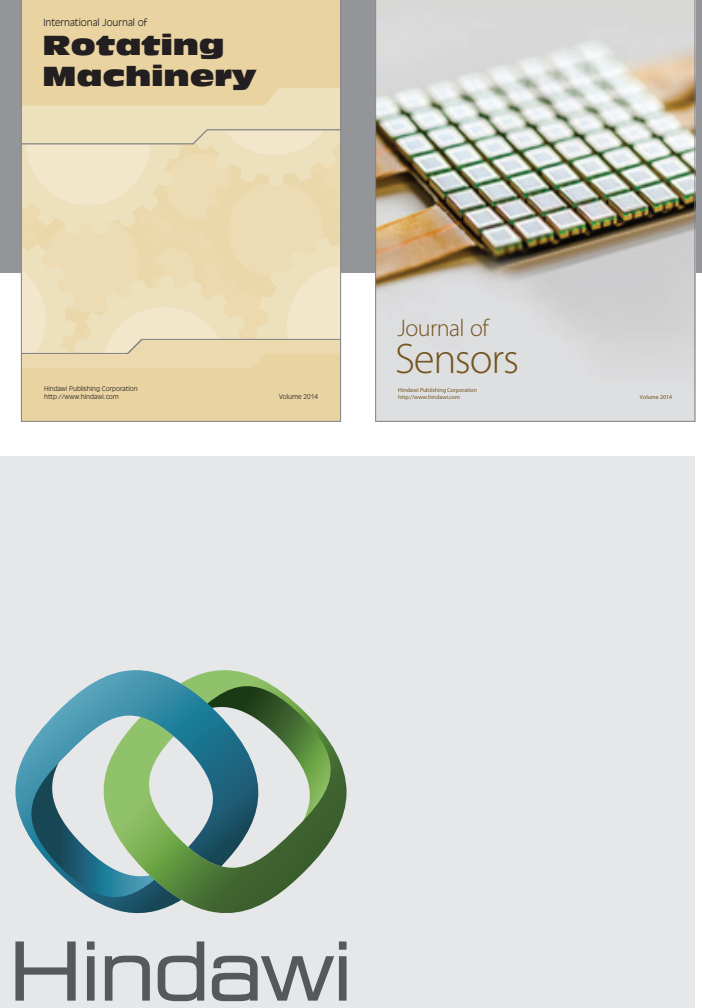

Submit your manuscripts at http://www.hindawi.com
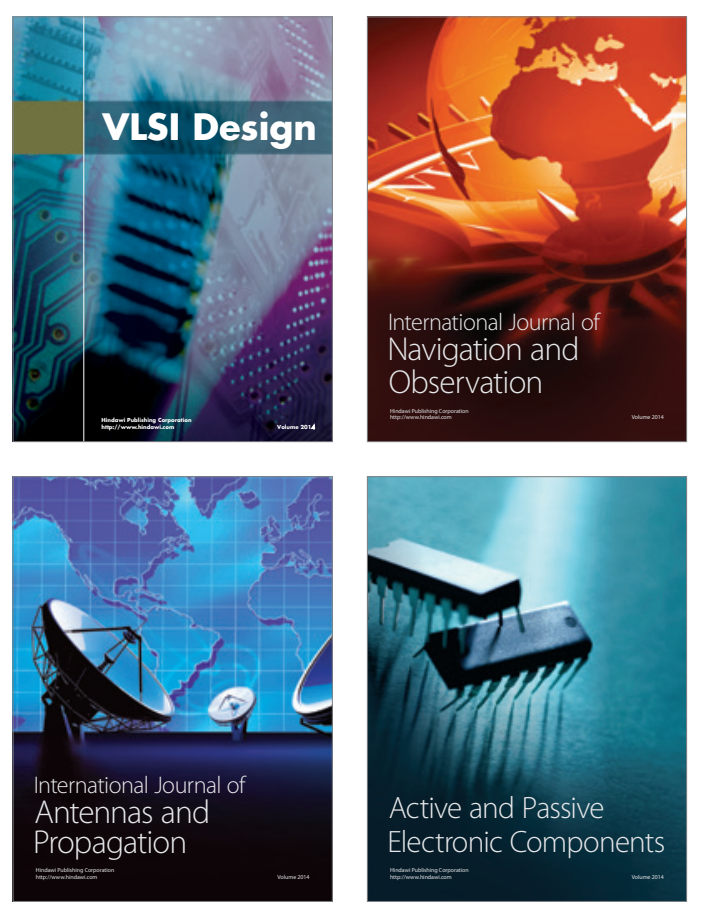
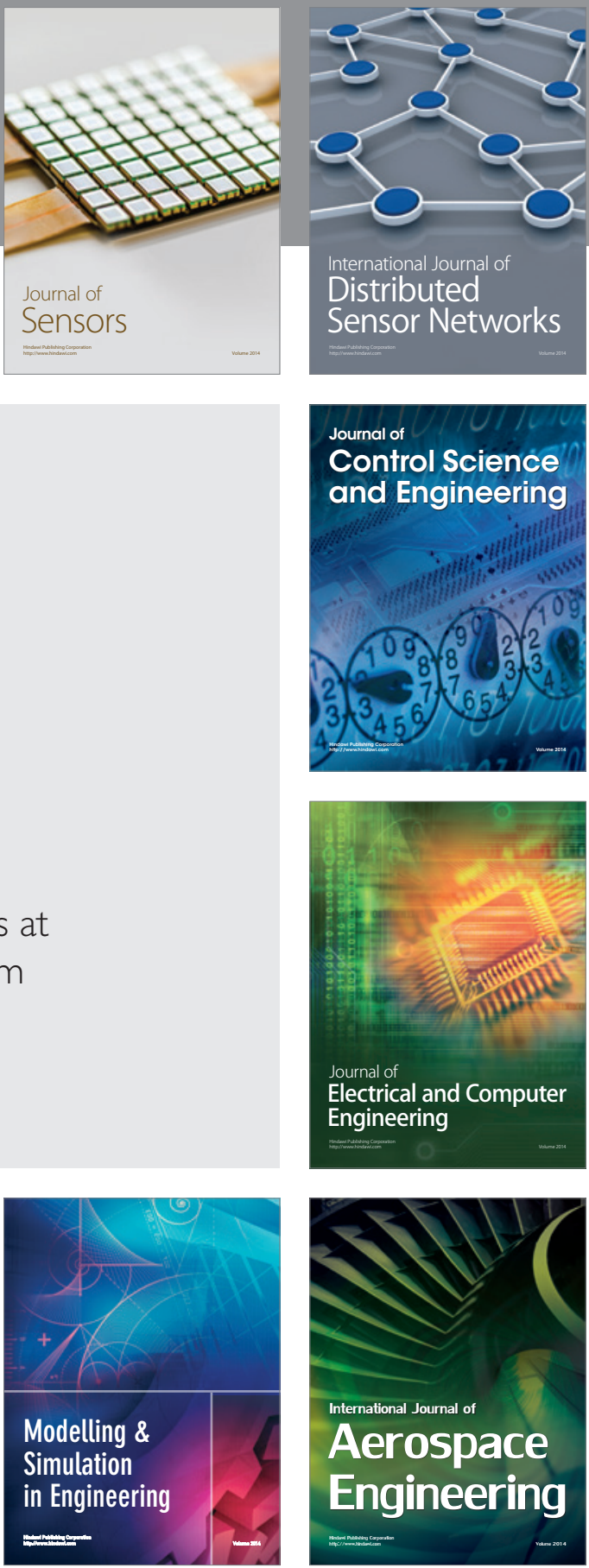

Journal of

Control Science

and Engineering
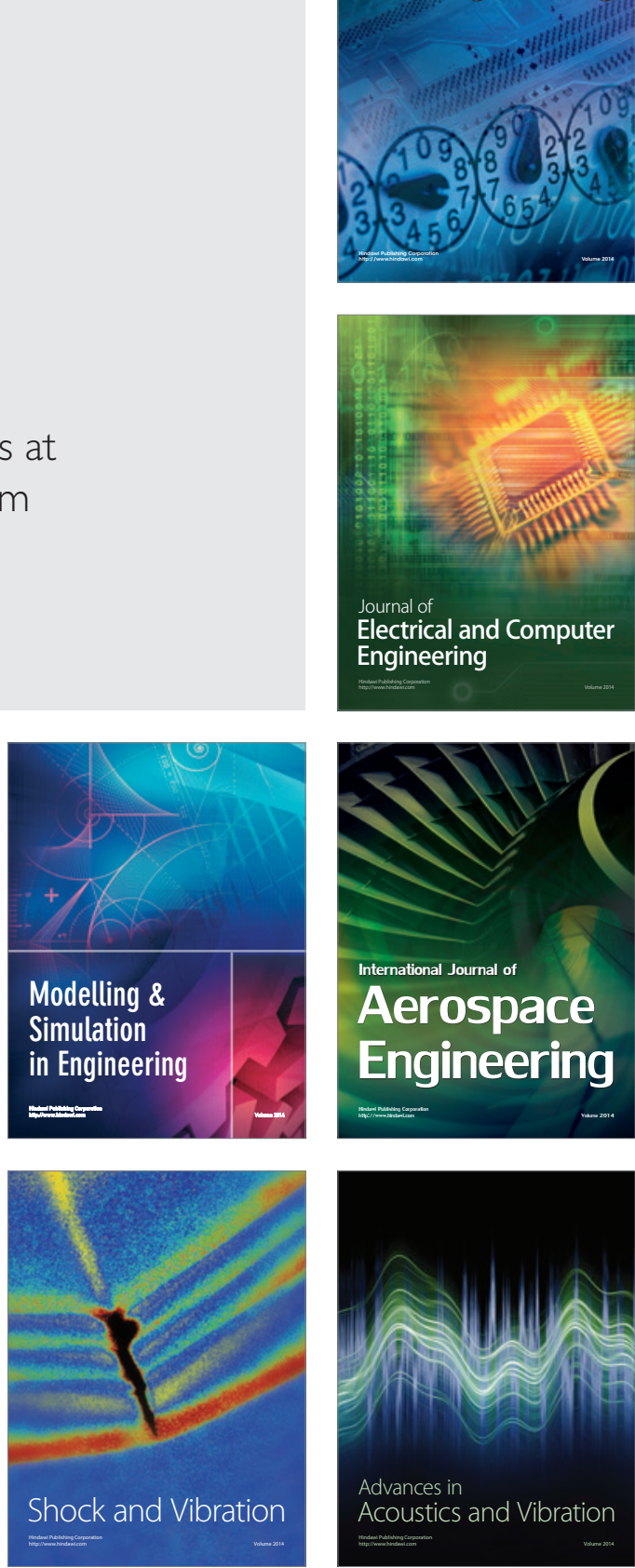